\title{
Does Partisan Conflict Predict a Reduction in US Stock Market (Realized) Volatility? Evidence from a Quantile-on-Quantile Regression Model ${ }^{\#}$
}

\author{
Rangan Gupta ${ }^{*}$, Christian Pierdzioch ${ }^{* *}$, Refk Selmi ${ }^{* * *}$ and Mark E. Wohar ${ }^{* * *}$
}

\begin{abstract}
Theory suggests that partisan conflict negatively affects the possibility of economic policy change, implying that financial markets tend to operate under lower policy risk. Given that stockreturn volatility measures risk, if the gridlock argument holds, stock-market volatility should be lower under divided than under a unified government. Using a partisan conflict index (PCI), we empirically confirm this theoretical argument for the U.S. stock market based on quantiles-based regressions. In particular, quantile-on-quantile regressions show that PCI tends to predict reduced volatility, with the effect being stronger at levels of volatility that are moderately low (i.e., below the median, but not at its extreme) for an increase in the predictor, especially with moderately low and high initial values (i.e., when PCI is at quantiles around the median).
\end{abstract}

Keywords: Partisan Conflict, Realized Volatility, Quantile Regressions. JEL Codes: C22, E60, G15.

\section{Introduction}

Bechtel and Füss (2008), using Fiorina's (1991) balancing model (dealing with the possibility of economic policy change under unified and divided government) have developed a simple model to demonstrate that under certain extreme conditions, the risk of policy change is reduced with a divided government (partisan conflict), since there is no alternative policy which all veto players prefer over the existing one. The result that lower policy uncertainty leads to lower macroeconomic volatility has also been theoretically derived more recently by Pastor and Veronesi $(2012$, 2013). One of the implications of this result, empirically verified for Germany over 1970 to 2005 by Bechtel and Füss (2008), is that higher partisan conflict is likely to dampen stock-market volatility, because with a divided government, stock markets can operate under lower policy risk. ${ }^{1}$

Given that stock market volatility is a key factor in asset allocation, portfolio optimization, and risk management (Rapach et al., 2008), the objective of this paper is to analyze whether a measure of the degree of partisan conflict in the US, based on an index developed by Azzimonti (2015), can help predict (realized) volatility of the S\&P500. In order to obtain the partisan conflict index (PCI), Azzimonti (2015) uses a semantic search approach to measure the

\footnotetext{
\# We would like to thank two anonymous referees for many helpful comments. Any remaining errors are solely ours.

* Department of Economics, University of Pretoria, Pretoria, 0002, South Africa. Email: rangan.gupta@up.ac.za.

** Department of Economics, Helmut Schmidt University, Holstenhofweg 85, P.O.B. 700822, 22008 Hamburg, Germany. Email: c.pierdzioch@hsu-hh.de.

*** University of Tunis, Campus Universitaire, Avenue 7 Novembre, 2092, Tunis, Tunisia; University of Pau, France. Email: s.refk@yahoo.fr.

**** Corresponding author. College of Business Administration, University of Nebraska at Omaha, 6708 Pine Street, Omaha, NE 68182, USA; School of Business and Economics, Loughborough University, Leicestershire, LE11 3TU, UK.Email: mwohar@unomaha.edu.

1 The link between stock-market developments and politics has also been studied extensively in the literature dealing with the implications of political cycles and the political orientation of the government for stock returns. See for example, Santa-Clara and Valkanov (2003), Döpke and Pierdzioch (2006), and Bohl et al. (2009), among others.
} 
frequency of newspaper coverage of articles reporting political disagreement about government policy (both within and between national parties) normalized by the total number of news articles within a given period. This data is available at a monthly frequency since January 1981, and with stock market data available at daily frequency, we can compute a model free-measure of volatility, that is, realized volatility (sum of daily squared returns over a month, see Andersen and Bollerslev, 1998).

We analyze the predictive ability of the PCI for realized stock-market volatility using standard Ordinary Least Squares (OLS) and two different quantile-based predictive regression approaches (standard quantile regressions and quantile-on-quantile [QQ] regressions), using data covering the period from January, 1981 to April, 2017. The advantage of the quantile regression approaches over conditional mean-based models is that the former can study the entire conditional distribution of volatility, i.e., they are inherently time-varying approaches capturing the various phases of volatility (low [lower quantiles], normal [median], high [higher quantiles]) of the US equity market. The QQ regression goes even a step further because it renders it possible to analyze the response of the entire conditional distribution of stock-market volatility simultaneously to various degrees political disagreement (as captured by the quantiles of the PCI) as well. To the best of our knowledge, this is the first paper to analyze the ability of the PCI to predict US stock-market volatility US using quantile regressions. ${ }^{2}$ The remainder of the paper is organized as follows: Section 2 outlines in brief the methodologies used, while Section 3 discusses the data and results, with Section 4 concluding the paper.

\section{Methodology}

In order to study the predictive ability of the PCI for the realized volatility (RV) of the S\&P500, we start with linear OLS regressions but then switch to quantile-regression approaches. The quantile regression approach (QRA), since its introduction by Koenker and Bassett (1978), has become a widely-studied modelling tool in economics and finance. The approach involves a set of regression curves that differ across different quantiles of the conditional distribution of the dependent variable, with the quantiles capturing various (time-varying) phases of the dependent variable (i.e., RV). Compared with a OLS linear regression or even non-linear regression methods, the quantile functions provide a more precise and accurate picture of the impact of covariates (in this case, lagged PCI) on the dependent variable (Koenker, 2005). The advantage of the QRA is its ability to recover information of tail dependence/predictability (i.e., upper and lower tails) beyond the median, which can be considered to capture the normal phase of the dependent variable.

A shortcoming of the standard QRA is its inability to capture dependence in its entirety. Specifically, even though the QRA estimates the heterogeneous response of RV to PCI at various points of the conditional distribution of the former, it overlooks the possibility that the nature (i.e. low, normal, or high levels) of PCI also could influence the way RV is predicted. Modeling this type of dependence requires a QQ approach. Using the QQ approach, we are able to model the quantile of RV as a function of the quantile of lagged PCI, so that the relationship

\footnotetext{
2 In somewhat related studies by Cheng et al. (2016) and Gupta et al. (forthcoming c), PCI has been shown to predict stock returns in- and out-of-sample, based on conditional mean-based linear and nonlinear models.

${ }^{3}$ Quantile regression was introduced in the seminal paper by Koenker and Bassett (1978). It is a generalization of median regression to other quantiles. The coefficients of the $\tau^{\text {th }}$ conditional quantile distribution are estimated as $\widehat{\beta}(\tau)=\arg \min \sum_{t=1}^{\tau}\left(\tau-1_{\left\{y_{t}<x_{t}^{\prime} \beta(\tau)\right\}}\right)\left|y_{t}-x_{t}^{\prime} \beta(\tau)\right|$, where the quantile regression coefficient $\beta(\tau)$ determines the connection between the vector of independent variables and the $\tau^{\text {th }}$ conditional quantile of the dependent variable, with $1_{\left\{y_{t}<x_{t}^{\prime} \beta(\tau)\right\}}$ being the usual indicator function.
} 
between these variables could vary at each point of their respective distributions. The QQ approach, thus, provides a more complete picture of dependence. We implement the QQ approach by (i) selecting a number of quantiles of lagged PCI, and, (ii), estimating the local effect these particular quantiles of the lagged PCI might have on the various quantiles of RV.

While there is also the triangular system of equations-based approach of Ma and Koenker (2006) for estimating QQ models, we use the single equation regression method of Sim and Zhou (2015) given that it can be easily estimated.

Let $\theta$ superscript denote the quantile of $\mathrm{RV}$. We first postulate a model for the $\theta$-quantile of $\mathrm{RV}$ as a function of its past lag $\mathrm{RV}_{\mathrm{t}-1}$, and one lag each of the PCI and the first principal component $(F)$ obtained from measures of output growth, inflation and short-term interest rate (discussed in detail in the data segment) to control for omitted variable bias, based on the suggetions of Engle and Rangel (2008), Rangel and Engle (2011), and Engle et al., (2013). Note that the lag-length of one based on the Schwarz Information Criterion (SIC). We have

$$
R V_{t}=\alpha^{\theta} R V_{t-1}+\beta^{\theta} P C I_{t-1}+\gamma^{\theta} F_{t-1}+\varepsilon_{t}^{\theta},
$$

where $\varepsilon_{t}^{\theta}$ is an error term that has a zero $\theta$-quantile. We allow the relationship function $\beta^{\theta}($. to be unknown, since we do not have a prior on how RV and PCI are interlinked. In order to examine the linkage between the $\theta$-quantile of RV and $\theta$-quantile of PCI, denoted by $P C I^{\tau}$, we linearize the function $\beta^{\theta}$ (.) by taking a first-order Taylor expansion of $\beta^{\theta}$ (.) around $P C I^{\tau}$, which yields the following:

$$
\beta^{\theta}\left(P C I_{t-1}\right) \approx \beta^{\theta}\left(P C I^{\tau}\right)+\beta^{\theta^{\prime}}\left(P C I^{\tau}\right)\left(P C I_{t-1}-P C I^{\tau}\right)
$$

Based on a study by Sim and Zhou (2015) study, we can redefine $\beta^{\theta}\left(P C I^{\tau}\right)$ and $\beta^{\theta^{\prime}}\left(P C I^{\tau}\right)$, respectively, as $\beta_{0}(\theta, \tau)$ and $\beta_{1}(\theta, \tau)$.

Equation (2) then can be re-written as follows:

$$
\beta^{\theta}\left(P C I_{t-1}\right) \approx \beta_{0}(\theta, \tau)+\beta_{1}(\theta, \tau)\left(P C I_{t-1}-P C I^{\tau}\right) .
$$

Next, we substitute equation (3) into equation (1) to obtain

$$
R V_{t}=\beta_{0}(\theta, \tau)+\alpha(\theta) \mathrm{RV}_{t-1}+\beta_{1}(\theta, \tau)\left(P C I_{t-1}-P C I^{\tau}\right)+\gamma(\theta) F_{t-1}+\varepsilon_{t}^{\theta},
$$

where we have defined $\alpha^{\theta} \equiv \alpha(\theta)$, with equation (4) applying to the the $\theta$ conditional quantile of RV. Unlike a standard conditional quantile function, the expression $\beta_{0}(\theta, \tau)+\beta_{1}(\theta, \tau)\left(P C I_{t-1}-P C I^{\tau}\right)$ captures the relationship between the $\theta$-quantile of RV and $\tau$-quantile of the lagged PCI, given that $\beta_{0}$ and $\beta_{1}$ are doubly indexed in $\theta$ and $\tau$. In other words, equation (4) captures the overall dependence structure between the RV and the lagged PCI through the dependence between their distributions.

\footnotetext{
4 The SIC criterion is known to select a parsimonious number of lags and, thereby, prevents overparameterization problems. Hurvich and Tsai (1989) examine the AIC and show that it is biased towards selecting an overparameterized model, while the SIC is asymptotically consistent. Complete details of the lag-length tests are available upon request from the authors.
} 
In order to estimate equation (4), we solve

$$
\min _{\beta_{0} \beta_{1}} \sum_{i=1}^{n} \rho_{\theta}\left[R V_{t}-\beta_{0}-\beta_{1}\left(P C I_{t-1}-P C I^{\tau}\right)-\alpha(\theta) R V_{t-1}-\gamma(\theta) F_{t-1}\right] K\left(\frac{F_{n}\left(P C I_{t-1}\right)-\tau}{h}\right)
$$

to obtain estimates $\hat{\beta}_{0}(\theta, \tau)$ and $\hat{\beta}_{1}(\theta, \tau)$, where the function $\rho_{\theta}$ is the tilted absolute value function that provides the $\theta$-conditional quantile of $\mathrm{RV}$ as the solution. Because we are interested in the effect exerted locally by the $\tau$-quantile of the lagged PCI, we use a Gaussian kernel $K($.$) to weight the observations in the neighbourhood of P C I^{\tau}$, based on a bandwidth $b$ $(=0.05$, following Sim and Zhou, 2015). The weights are inversely related to the distance of $P C I_{t-1}$ from $P C I^{\tau}$, or more conveniently, the distance of the empirical distribution function

$F_{n}\left(P C I_{t-1}\right)=\frac{1}{n} \sum_{k=1}^{n} I\left(P C I_{k}<P C I_{t-1}\right)$

from $\tau$, where $\tau$ is the value of the distribution function that corresponds to $P C I^{\tau}$.

\section{Data and Results}

The dataset we analyze covers the monthly period 1981:01-2017:04 (i.e., 436 observations), and incorporates two main variables: the realized volatility (RV) of the S\&P500 and the news-based index of partisan conflict (PCI) introduced by Azzimonti (2015), and a control variable in the form of a factor derived from the first principal component of output growth, inflation and a measure of short-term interest rate. The RV is defined as the daily sum over a month of the squares of the continuously compounded S\&P 500 returns. ${ }^{5}$ The PCI index is log-transformed, ${ }^{6}$ and tracks the degree of political disagreement among US politicians at the federal level by measuring the frequency of newspaper articles in major US newspapers (Washington Post, New York Times, Los Angeles Times, Chicago Tribune, and Wall Street Journal) reporting disagreement in a given month. ${ }^{7}$ The start and end date of the sample is purely driven by the availability of data on the PCI. Figure A1 plots the RV and the natural logarithm of the PCI index (PCI), while Table A1 reports summary statistics of these two variables (see the end of the paper). The non-normality due to positive skewness and excess kurtosis (i.e., heavy right-tails), provides a strong motivation to look at quantiles-based approaches, and in particular the QQ regression. Note that, for the construction of the factor, we use industrial production growth, consumer price index (month-on-month inflation), both derived from the FRED database of the Federal Reserve Bank of St. Louis, and Krippner's (2013) shadow short rate as a measure of short-term interest rate, given that our sample period involves the zero lower bound (ZLB) situation. ${ }^{8}$ Krippner (2013) obtains the shadow short rate as a proxy for the short-term policy rate based on a multi-factor model of the term-structure. The shadow short rate is considered to be a better measure of the monetary policy stance of the Federal Reserve instead of the Federal

\footnotetext{
5 The measure that we consider is the classical estimator of realized volatility, i.e., the sum of squared daily returns (Andersen and Bollerslev, 1998), expressed as: $R V_{t}=\sum_{i=1}^{M} y_{t, i}^{2}$, where $y_{t, i}$ is the daily $M \times 1$ return vector and $i=1, \ldots, M$ the number of daily returns. The daily S\&P500 nominal stock-price data from $2^{\text {nd }} J a n u a r y ~ 1981$ to $28^{\text {th }}$ April 2017 is obtained from the Datastream database maintained by Thomson Reuters.

${ }^{6}$ Standard unit root tests reveal that the natural logarithm of the PCI is stationary. The details of these tests are available upon request from the authors.

7 Data and further details are available at: https://www.philadelphiafed.org/research-and-data/real-timecenter/partisan-conflict-index.

8 The data can be downloaded from: https://www.rbnz.govt.nz/research-and-publications/researchprogramme/additional-research/measures-of-the-stance-of-united-states-monetary-policy.
} 
Funds rate, especially during the post-financial crisis period, where unconventional measures of monetary policy were adopted. ${ }^{9}$

The OLS estimate of the lagged PCI on RV was found to be -0.0012 , i.e., negative as suggested by theory, but this coefficient was not significant even at the 10 percent level of significance, with the $p$-value being 0.2416 . The QRA is expected to provide a more complete and robust estimates in any case. To further validate the use of the quantiles-based approaches, which are essentially nonlinear, we conducted the Brock et al., (1996) test of nonlinearity on the residuals recovered from the OLS model $\left(R V_{t}=\beta_{0}+\beta_{1} P C I_{t-1}+\alpha R V_{t-1}+\gamma F_{t-1}+\varepsilon_{t}\right)$. The results are reported in Table A2 and provide overwhelming evidence of nonlinearity, as we reject the null of i.i.d. at all possible dimensions at a 1 percent level of significance. In other words, results from the linear model cannot be deemed reliable in any case. ${ }^{10}$ As can be seen from Figure 1, which reports the impact of the lagged PCI at the various quantiles of RV, the relationship is consistently negative over the entire conditional distribution of $\mathrm{RV}$, with the effect getting stronger beyond the median. More precisely, the effect is statistically significant at the 5 percent level over the quantile range from 0.20 to $0.75,{ }^{11}$ i.e., barring the extreme ends of the conditional distribution of RV. In sum, consistent with economic theory, the lagged PCI does have a negative impact on $\mathrm{RV}$, with the effect being significant except at the extreme low and high quantiles.

Figure 1. Quantiles-Based Response of RV to Lagged PCI (QRA)

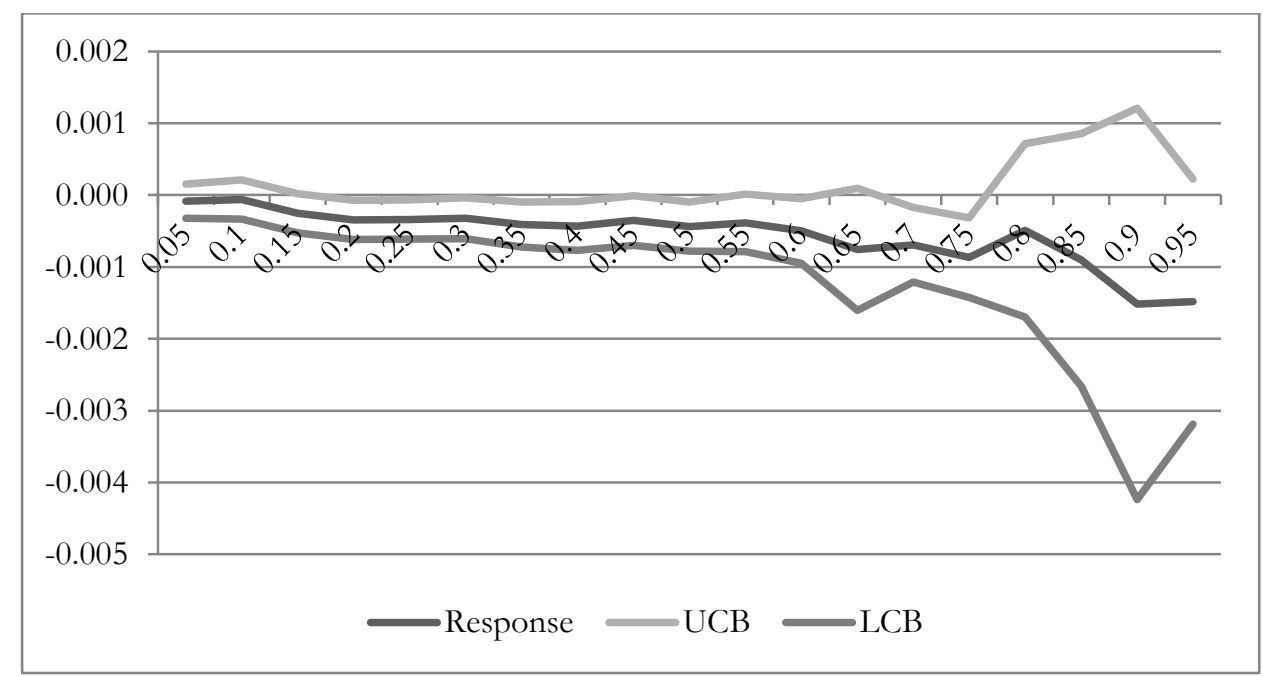

Note: UCB and LCB are upper and lower 95 percent confidence bands respectively.

\footnotetext{
${ }^{9}$ Based on the suggestions of an anonymous referee, we also constructed the principal component by including the University of Michigan's Consumer Sentiment Survey Index, and also the news-based economic policy uncertainty index of Baker et al., (2016). Our results (as discussed later) were, however, qualitatively the same when we constructed the principal component based on the industrial production growth, CPI inflation and the shadow short rate only. Complete detail of this analysis is available upon request from the authors.

${ }^{10}$ In addition, we tested whether the response of the RV to the lagged PCI is statistically different at various quantiles. The associated test of Koekner and Xiao (2002) overwhelmingly rejected the null hypothesis of slope equality across the quantiles of RV at the highest level of significance. This result (along with results of the BDS test, see Table A2) provides strong statistical reasons for the usage of quantiles-based models over a standard OLS regression. Complete details of the Koekner and Xiao (2002) test are available upon request from the authors. However, it must be noted, there is no available comparable test in the QQ framework, which would allow us to analyze whether the PCI at different quantiles have statistically different impact on RV.

11 The effect, although negative, was not significant at the quantiles 0.55 and 0.65 at the 5 percent level of significance. However, at the ten percent level of significance, the response is significant over the entire quantile range of 0.15 to 0.75 .
} 
Next, we turn to the results from the QQ estimation. Figure 2(a) plots the response surface of RV to lagged PCI for various combinations of the quantiles $(\theta$ and $\tau)$ of the dependent and the independent variables. The figure shows that a higher lagged PCI predicts a lower RV, but the strength of this effect depends on the quantiles of the latter. For a given quantile of RV, the negative impact of the lagged PCI tends to vary, not necessarily in a systematic fashion, over the quantiles of the predictor. What we observe, however, is that the predictability of LPCI is stronger at its moderately lower and moderately upper quantiles around the median. That is, an increase in PCI has a relatively stronger negative impact on RV if the initial level of LPCI from which it is increasing is in fact moderately low or moderately high. However, unlike the QAR results in Figure 1, the strongest impact of lagged PCI is found at quantiles of RV below the median. This result suggests that the negative impact of lagged PCI is felt strongly when it increases around its normal mode (i.e., median), given that RV itself is relatively low. ${ }^{12}$ The predictive value of PCI, if it has settled at an extreme low or high state, is weaker (than around the median) when RV itself is exceptionally high. In sum, the negative impact of lagged PCI on $\mathrm{RV}$ is more pronounced when the former increases from an initial value that is around the median, with the RV being moderately lower than its normal state. ${ }^{13}$ More importantly, unlike the QRA, as shown in Figure 2(b), the responses of the conditional distribution of RV are consistently significant across the various quantiles of lagged PCI based on the QQ model. The overwhelming evidence of statistical significance derived under the QQ framework highlights the importance of this approach over a QRA model, especially given the fact that PCI itself has a non-normal distribution. Intuitively, our results tend to imply that information on the changes in partisan conflict is of relatively lesser value to investors in predicting movements of market volatility, especially if partisan conflict is already exceptionally low or high. However, when partisan conflict changes around its normal levels, it seems to send out relatively clearer signal to investors in terms of the possible reduced uncertainty in the economy and hence, its likely negative effect on volatility. However, for this to happen, market volatility should be low in general. The fact that market volatility at its upper end is not affected strongly by partisan conflict is most likely due to the fact that above normal market volatility in itself is a sign of higher uncertainty, and also there is some empirical evidence (see for example, Balcilar and Demirer (2015)) that agents tend to herd when market volatility is high, and hence information from the movements in partisan conflict could possibly of relatively lesser value. ${ }^{14}$

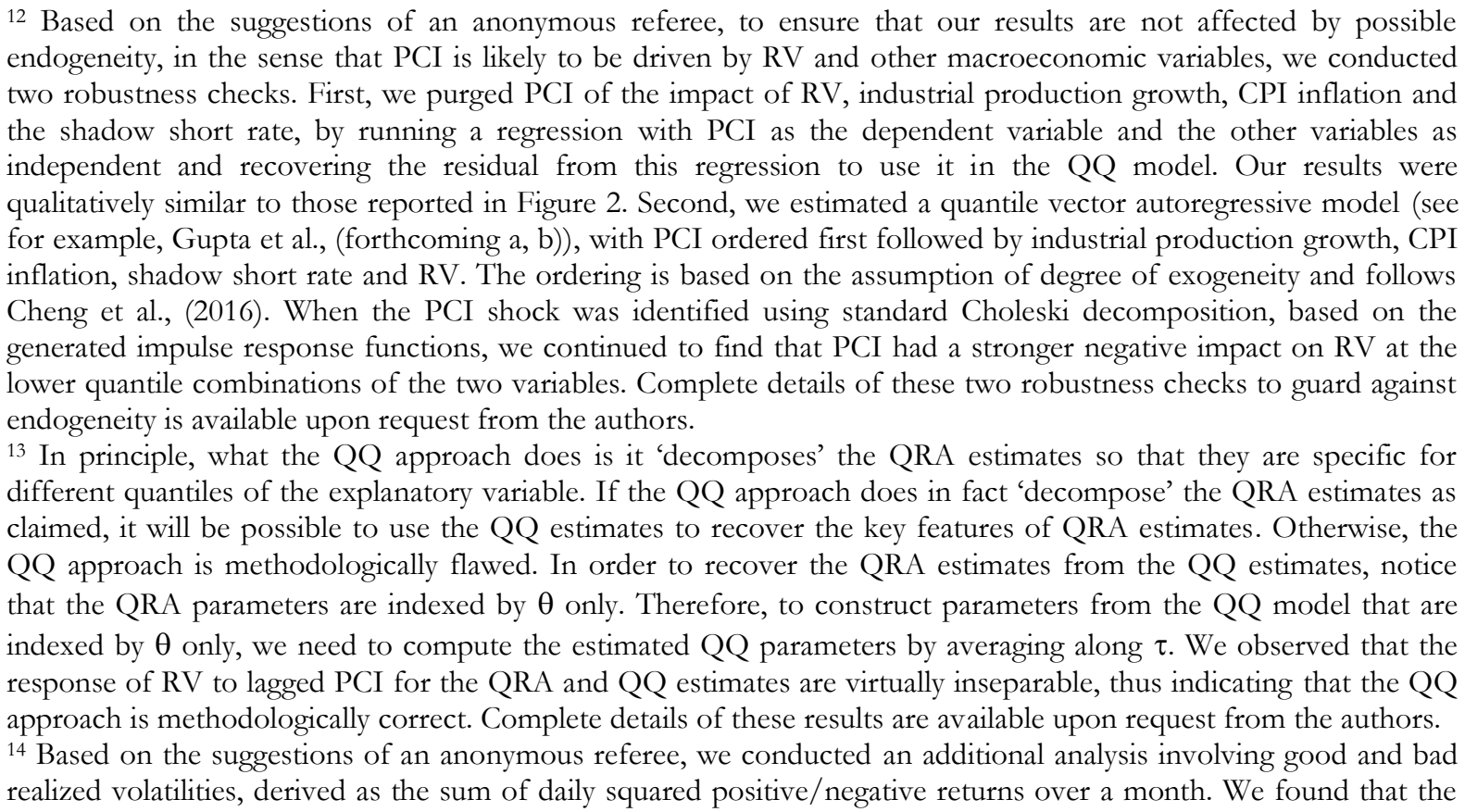


Figure 2(a). Quantiles-Based Response of RV to Quantiles of Lagged PCI (QQ Model)

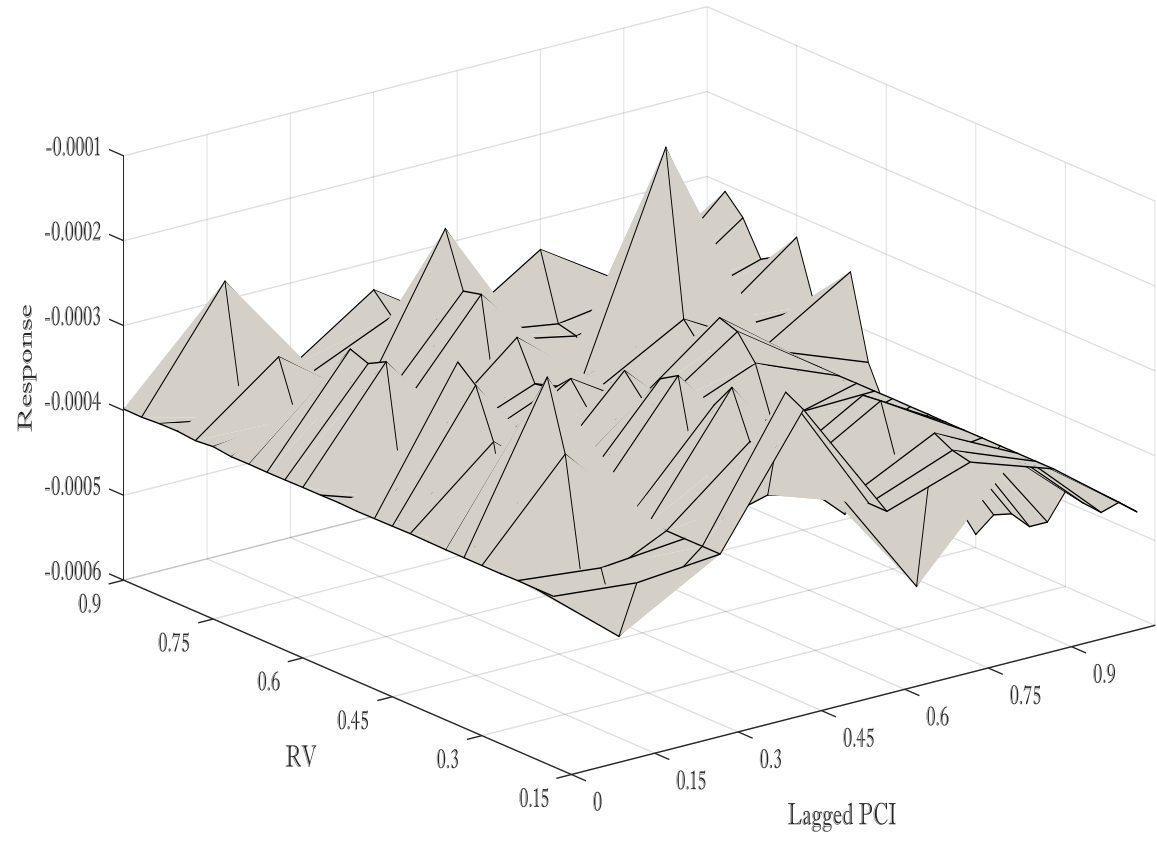

Figure 2(b). Quantiles-Based $t$-statistics of RV to Quantiles of Lagged PCI (QQ Model)

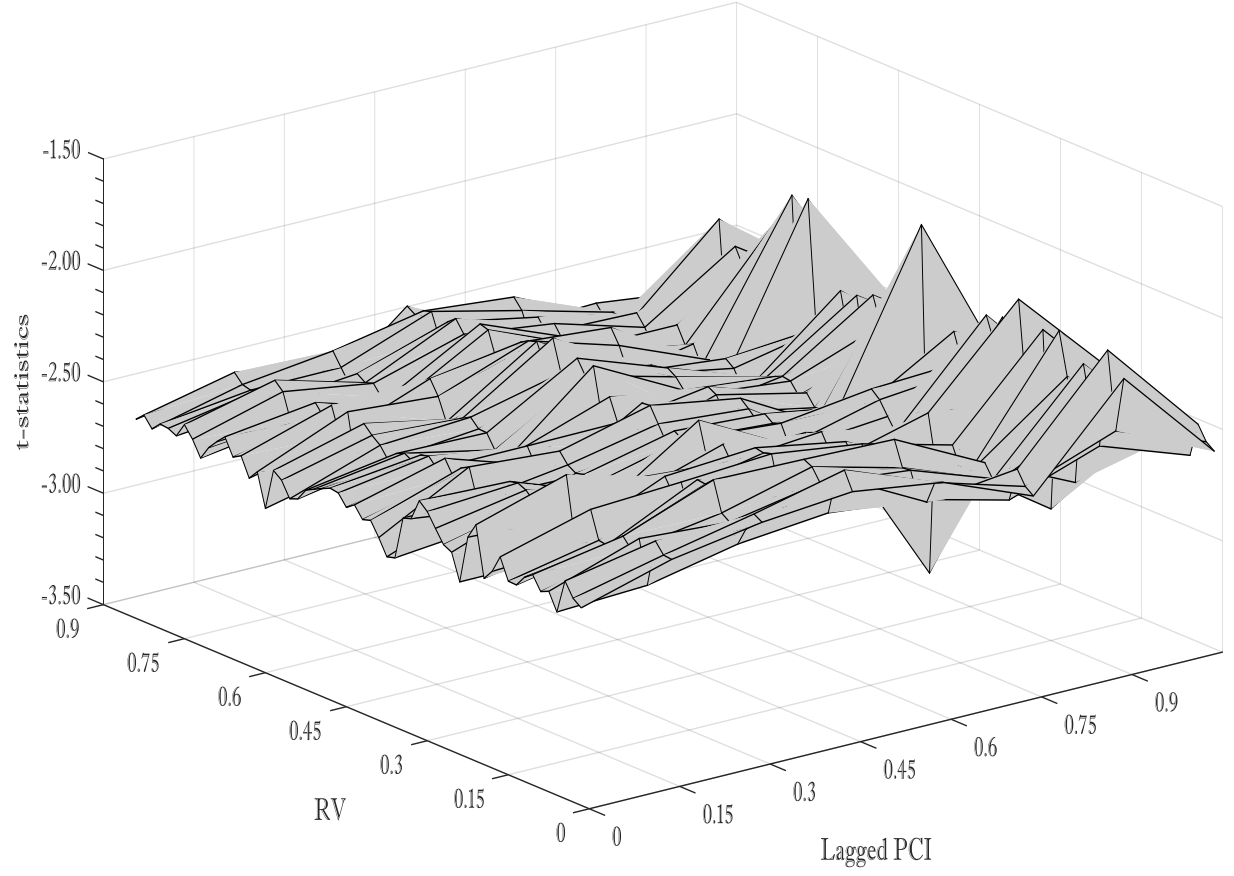

negative effect of lagged PCI is stronger and significant for good volatility across the quantiles of the two variables under consideration. Taking this result into account, we can conclude that it is the good realized volatility that tends to drive the results for the overall realized volatility. Complete details of these results are available upon request from the authors. 
Note that, our empirical results support the theoretical line of reasoning of Bechtel and Füss (2008), that higher partisan conflict decreases economic policy uncertainty, or at least causes market agents to perceive a possible reduction in economic policy uncertainty, and hence, a fall in market volatility. However, as observed in Azzimonti (2015), the relationship between partisan conflict and policy uncertainty might not be monotonic. Heightened partisan conflict could raise policy uncertainty, which in turn, could potentially increase stock market volatility. In fact, the unconditional correlation between PCI and the economic policy uncertainty (EPU) index developed by Baker et al. (2016) is positive found to be positive by Azzimonti (2015). ${ }^{15}$ Given this, we wanted to delve into this issue further, and analyze whether the impact of PCI on RV is in fact contingent on the state of the macroeconomy, i.e., whether the PCI affects RV differently during expansions and recessions. ${ }^{16}$ For this purpose, based on the NBER recession dates (available at: http://www.nber.org/cycles.html), we create two dummy variables, which takes the value of 1 when it is recession or expansion and zero when it is expansion or recession. Then we multiply these two dummies with the PCI variable to obtain a PCI corresponding to expansions and recessions, which in turn, are used to conduct the QQ estimation, with the principal component as an additional control variable. Interestingly, as indicated in Figure 3(a) and 3(b), we find that the PCI during expansions has a significant negative impact on RV, while the PCI during recession has a positive impact on $\mathrm{RV}$, with the effect in the latter case being only significant at higher quantiles of both the dependent and the independent variables (see Figures $\mathrm{A} 2$ and $\mathrm{A} 3$ in the Appendix of the paper, which plots the $t$-statistics from the QQ model during expansions and recessions). So our results tend to suggest that, the overall negative impact of PCI on RV is driven by the effect of the former during expansions, since expansions are more frequent than recessions. Intuitively, when the economy is performing well and there is increased partisan conflict, agents believe that economic policies are less likely to be changed, and hence, there is a reduction in policy uncertainty, which in turn reduces market volatility. It may also be noted, that during expansion, policy uncertainty is likely to be lower in any case than during recessions (Gupta et al., forthcoming a). But, during recessions, increased partisan conflict, especially if it is quite high, is likely to result in a policy change and volatility tends to rise, as a result of increased policy uncertainty.

\footnotetext{
${ }^{15}$ We would like to thank the second anonymous reviewer for pointing this out to us.

${ }^{16}$ We would like to thank the first anonymous reviewer for encouraging us to take this route.
} 


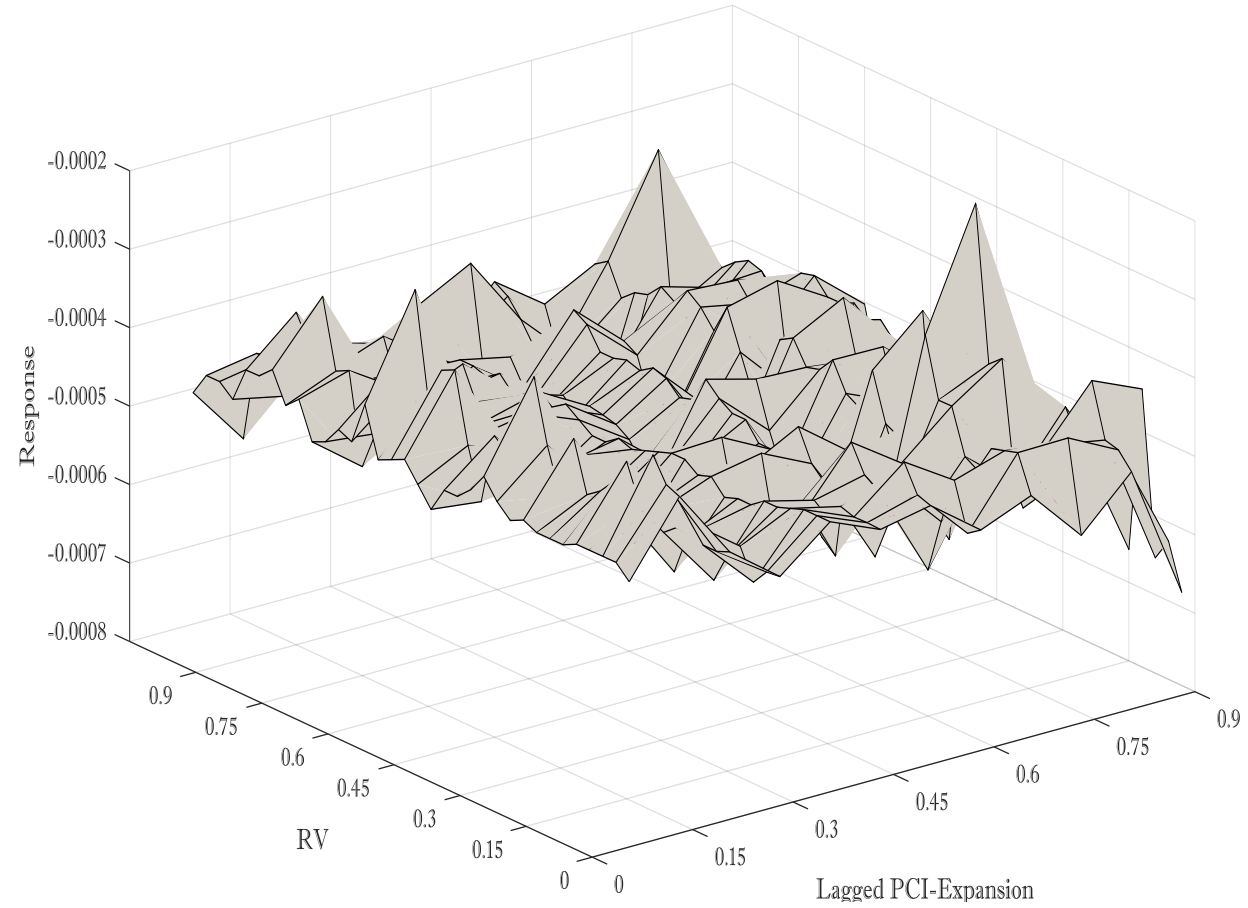

Figure 3(b). Quantiles-Based Response of RV to Quantiles of Lagged PCI during Recessions (QQ Model)

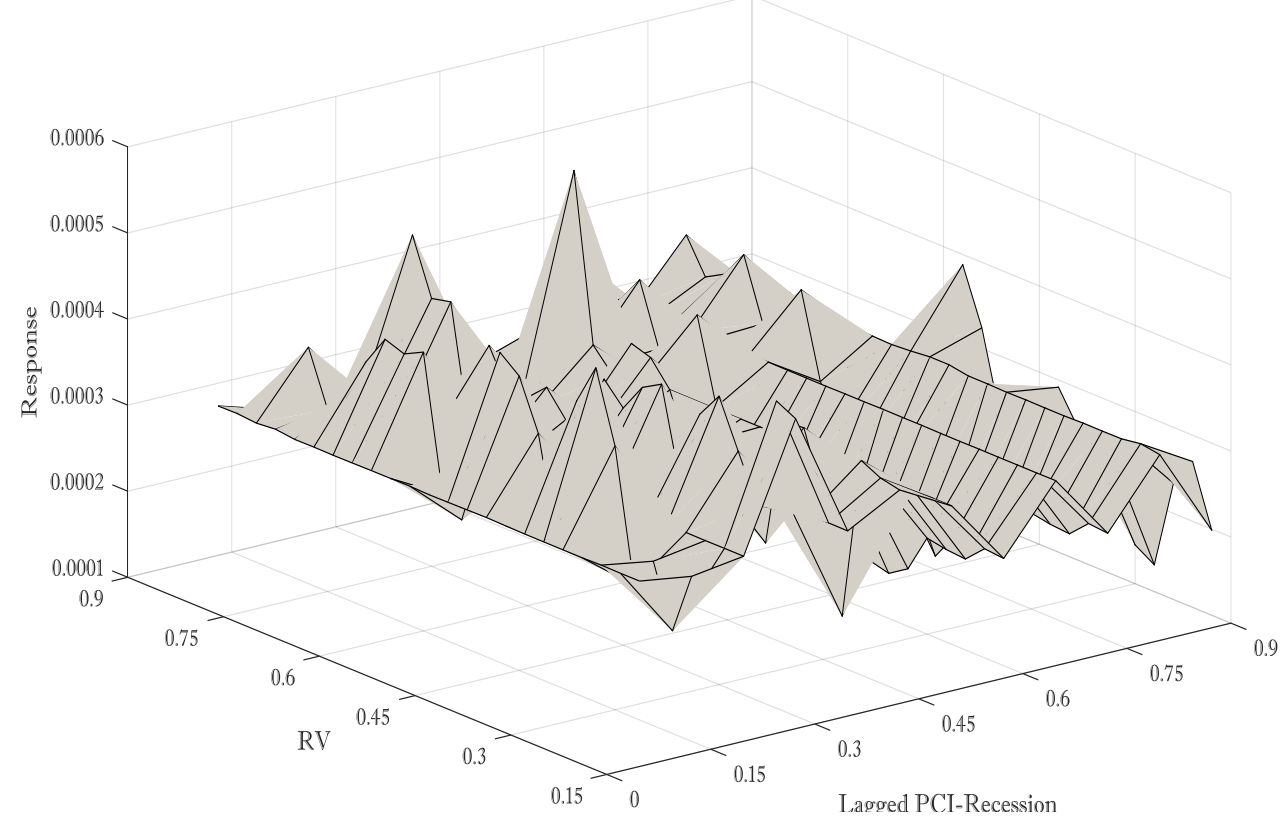

\section{Conclusion}

Theory suggests that partisan conflict negatively affects the possibility of economic policy change. Because divided government reduces the likelihood of economic-policy change, financial markets can operate under lower policy risk in times of higher partisan conflict. Given that stock market volatility measures risk in financial markets, if the gridlock argument applies higher partisan conflict should reduce stock-market volatility. The objective of this research has been to empirically test this prediction of economic theory using a recent monthly index of US partisan conflict as developed by Azzimonti (2015). With stock-market data available at a daily frequency, we have computed a model free-measure of volatility (realized volatility, RV) and have analyzed 
the predictive value of the partisan conflict index (PCI) over the period from January, 1981 to April, 2017, using quantiles-based predictive regressions. We started off with a standard linear OLS regression, which picked up a negative but insignificant relationship between the lagged PCI and RV. With tests of nonlinearity showing that the linear model is misspecified, we then moved on to quantiles-based models. Results for a standard quantile regression have shown that the lagged PCI predicts a significant lower RV barring the extreme ends of the conditional distribution of the latter. We then estimated quantile-on-quantile regressions, which have added another layer of information to this result by showing that the negative impact of lagged PCI on $\mathrm{RV}$ is more pronounced when the former increases from an initial value that is generally moderately low or high, i.e., around the median. Investors can learn from this result that that increases in partisan conflict reduce the risk of stock-market investments, with the reduction being particularly strong when volatility is moderately low, given that the increase in partisan conflict happened around its normal state. Moreover, unlike standard quantile regressions, our results based on quantile-on-quantile model is consistently significant over the quantiles of both the dependent and independent variables, thus highlighting the importance of looking at the entire distributions of these variables. Finally, we also show that our results are dependent on whether the economy is in recession or expansion, with the negative relationship between RV and PCI holding only when the economy is booming.

As part of future research, we could extend our analysis to check whether US partisan conflict can in fact also predict a reduction in volatility of other developed, as well as, developing stock markets. In addition, it would also be interesting to see if our results of in-sample predictability also tend to hold out-of-sample in a full-fledged forecasting exercise, since the former does not necessarily guarantee the latter (Rapach and Zhou, 2013). Moreover, given our empirical result that the impact of partisan conflict on market volatility is state-specific, it would make sense to develop a theoretical framework that accommodates for such a finding.

\section{References}

Andersen T.G., and Bollerslev T. (1998). Answering the skeptics: yes, standard volatility models do provide accurate forecasts. International Economic Review, 39 (4), 885-905.

Azzimonti, M. (2015). Partisan conflict and private investment. NBER Working Paper No. w21273.

Baker, S., Bloom, N., and Davis, S. (2016). Measuring economic policy uncertainty. Quarterly Journal of Economics, 131, 1593-1636

Balcilar, M., and Demirer, R. (2015). Effect of Global Shocks and Volatility on Herd Behavior in an Emerging Market: Evidence from Borsa Istanbul. Emerging Markets Finance and Trade, 51(1), 140-159.

Bechtel, M.M., and Füss, R. (2008). When investors enjoy less policy risk: Divided government, economic policy change, and stock market volatility in Germany, 1970-2005. Swiss Political Science Review 14(2), 287-314.

Bohl, M.T., Döpke, J., and Pierdzioch, C. (2008). Real-Time forecasting and political stock market anomalies: Evidence for the United States. Financial Review 43, 323-335.

Brock, W. A., Scheinkman, J. A., Dechert, W. D., and LeBaron, B. (1996). A test for independence based on the correlation dimension. Econometric Reviews 15(3), 197-235.

Cheng, C.-H. J., W. A. Hankins, and C.-W. J. Chiu (2016). Does US partisan conflict matter for the Euro area? Economics Letters 138, 64-67.

Döpke, J., and Pierdzioch, C. (2006). Politics and the stock market: Evidence from Germany. European Journal of Political Economy 22, 925-943. 
Engle, R.F., and Rangel, J.G. (2008). The Spline-GARCH Model for Low-Frequency Volatility and Its Global Macroeconomic Causes. Review of Financial Studies 21(3), 1187-1222.

Engle, R.F., \& Ghysels, E., and Sohn, B. (2013). Stock Market Volatility and Macroeconomic Fundamentals. The Review of Economics and Statistics 95(3), 776-797.

Fiorina, M. (1991). Divided government in the States. Political Science and Politics 24(4), 646-50.

Gupta, R., Lau, C.K.M., and Wohar, M.E. (Forthcoming a). The Impact of US Uncertainty on the Euro Area in Good and Bad Times: Evidence from a Quantile Structural Vector Autoregressive Model. Empirica.

Gupta, R., Lau, C.K.M., Miller, S.M., and Wohar, M.E. (Forthcoming b). U.S. Fiscal Policy and Asset Prices: The Role of Partisan Conflict. International Review of Finance.

Gupta, R., Muteba Mwamba, J.W., and Wohar, M.E. (Forthcoming c). The role of partisan conflict in forecasting the U.S. equity premium: A nonparametric approach. Finance Research Letters. (forthcoming)

Hurvich, C.M. and Tsai, C.-L. (1989). Regression and Time series model selection in Small samples. Biometrika 76, 297-307.

Koekner, R. and Xiao, Z (2002). Inference on the quantile regression process. Econometrica 70(4), 1583-1612.

Krippner, L. (2013). A Tractable Framework for Zero Lower Bound Gaussian Term Structure Models. Discussion Paper, Reserve Bank of New Zealand, 2013/02.

Ma, L. and Koenker, R. (2006). Quantile regression methods for recursive structural equation models. Journal of Econometrics 134, 471-506.

Pastor, L., and Veronesi, P. (2012). Uncertainty about government policy and stock prices. Journal of Finance 67, 1219--1264.

Pastor, L., and Veronesi, P. (2013). Political uncertainty and risk premia. Journal of Financial Economics 110, 520--545.

Rangel, J.G., and Engle, R.F. (2011). The Factor-Spline-GARCH Model for High and Low Frequency Correlations. Journal of Business \& Economic Statistics 30(1), 109-124.

Rapach, D.E., Strauss, J.K., and Wohar, M.E. (2008). Forecasting stock return volatility in the presence of structural breaks, in Forecasting in the Presence of Structural Breaks and Model Uncertainty, in David E. Rapach and Mark E. Wohar (Eds.), Vol. 3 of Frontiers of Economics and Globalization, Bingley, United Kingdom: Emerald (May 2008), pp. 381-416.

Rapach, D. E., \& Zhou, G. (2013). Forecasting stock returns. Handbook of Economic Forecasting, 2(Part A), Graham Elliott and Allan Timmermann (Eds.), Amsterdam: Elsevier, 328-38.

Santa-Clara, P. and Valkanov, R. (2003). The presidential puzzle: Political cycles and the stock market. Journal of Finance 58, 1841-1872.

Sim, N. and Zhou, A. (2015). Oil prices, US stock return, and the dependence between their quantiles. Journal of Banking and Finance 55, 1-8. 


\section{Appendix:}

Figure A1. Data Plots
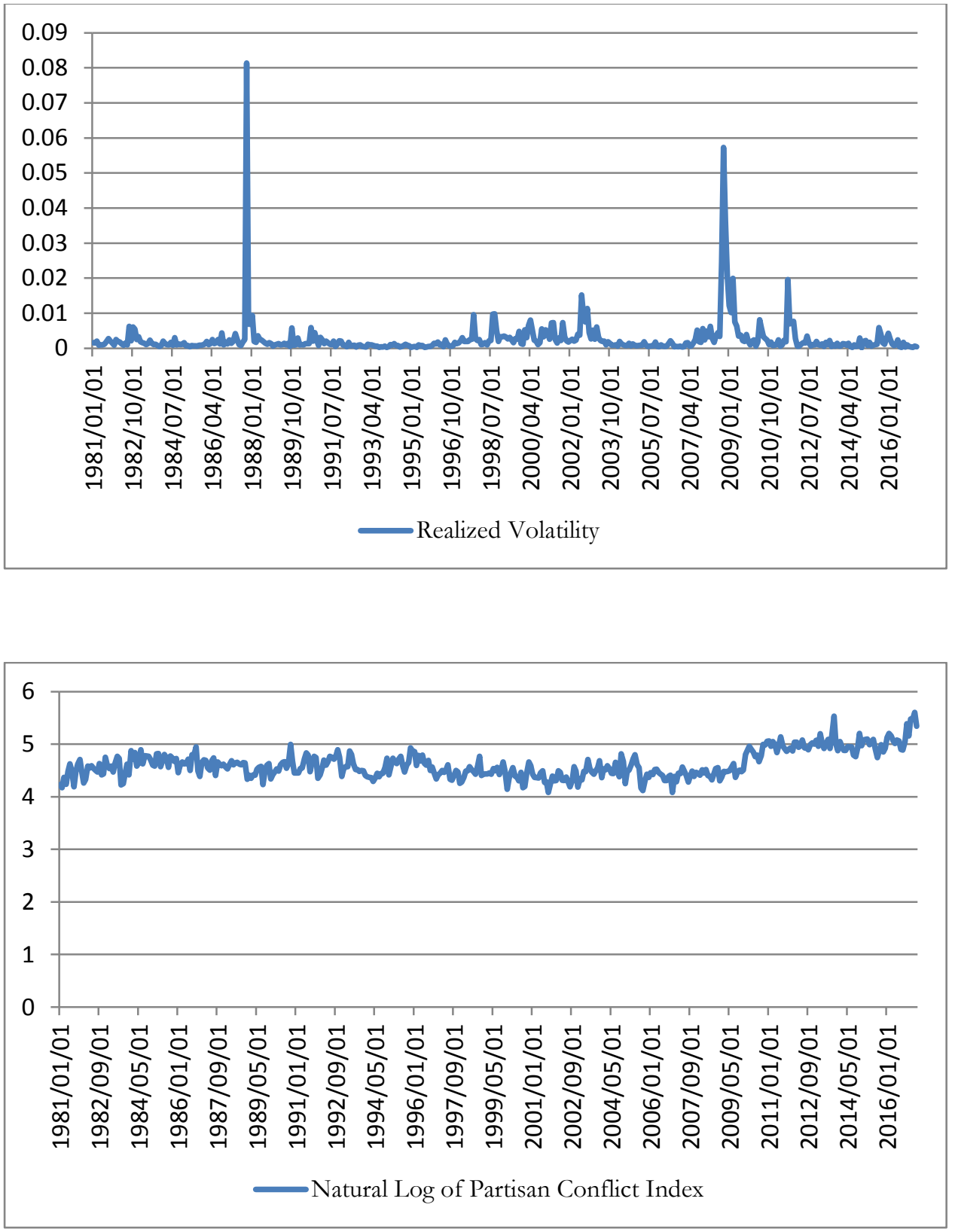
Figure A2. Quantiles-Based $t$-statistics of RV to Quantiles of Lagged PCI during Expansions (QQ Model)

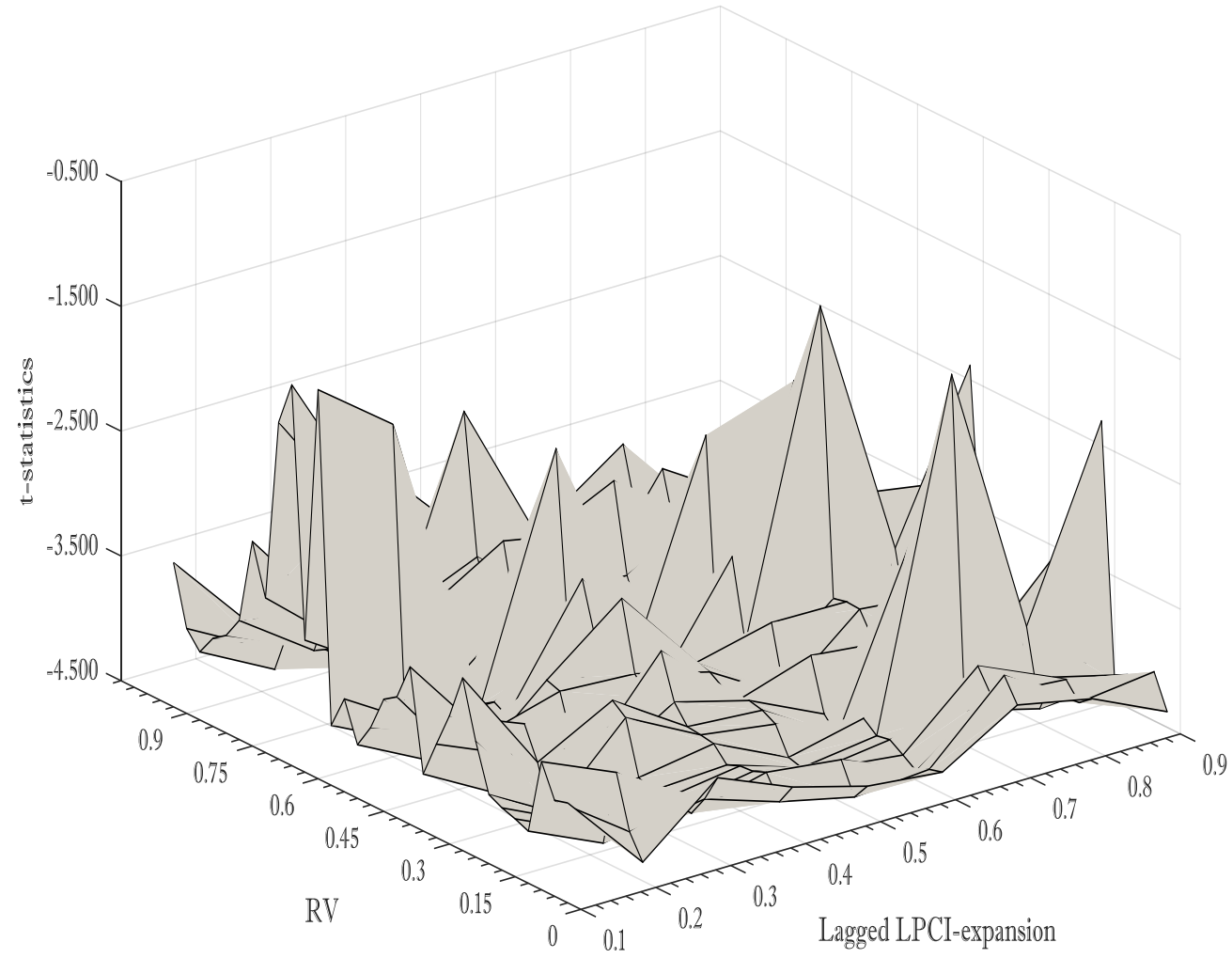

Figure A3. Quantiles-Based $t$-statistics of RV to Quantiles of Lagged PCI during Recessions (QQ Model)

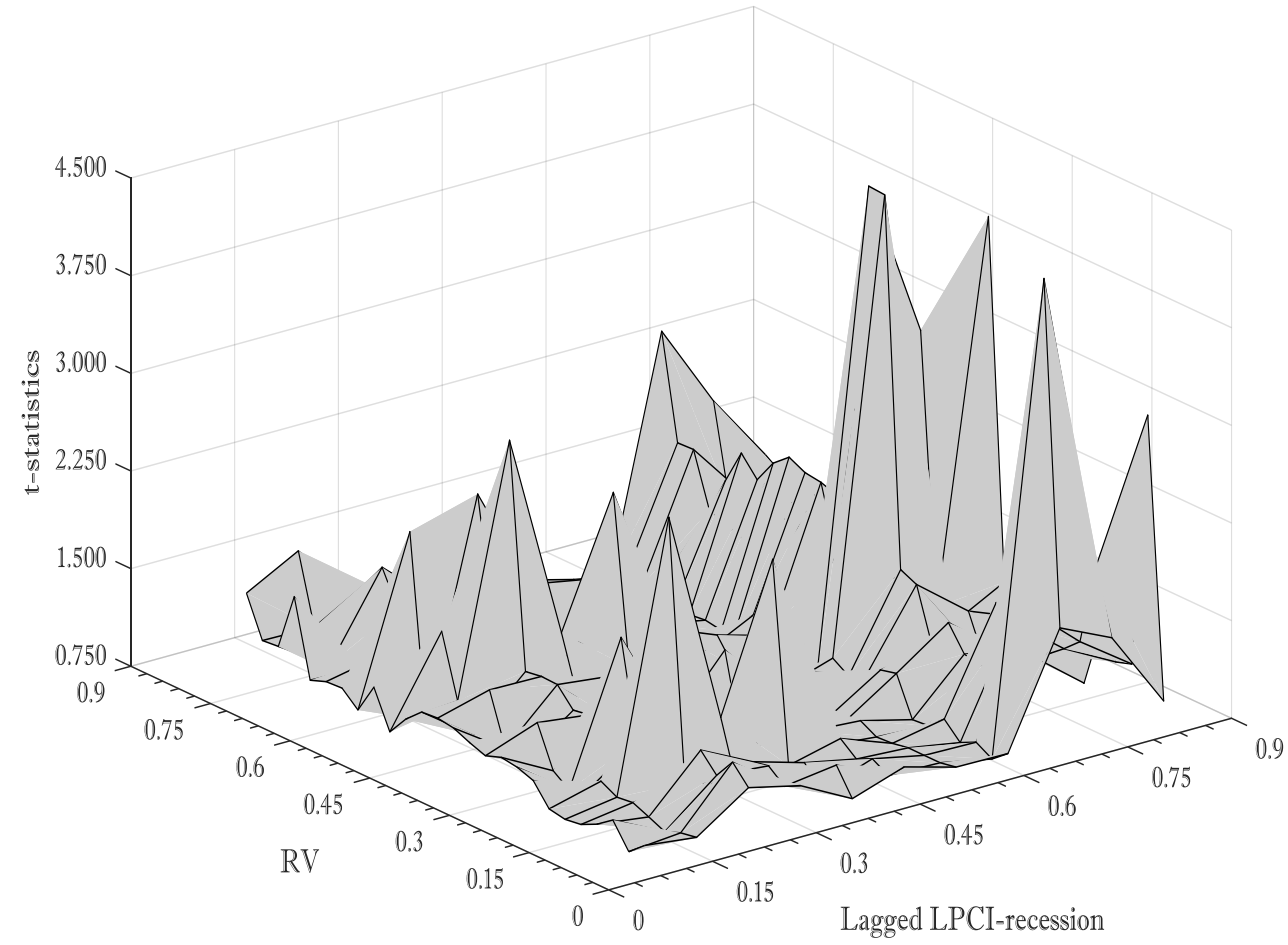


Table A1. Summary Statistics

\begin{tabular}{|c|c|c|}
\hline & \multicolumn{2}{|c|}{ Variable } \\
\hline Statistic & $\begin{array}{l}\text { Realized } \\
\text { Volatility }\end{array}$ & $\begin{array}{l}\text { Natural } \\
\text { Log of } \\
\text { Partisan } \\
\text { Conflict } \\
\text { Index }\end{array}$ \\
\hline Mean & 0.0026 & 4.6174 \\
\hline Median & 0.0014 & 4.5687 \\
\hline Maximum & 0.0814 & 5.6032 \\
\hline Minimum & 0.0002 & 4.0807 \\
\hline Std. Dev. & 0.0056 & 0.2557 \\
\hline Skewness & 9.4755 & 0.7699 \\
\hline Kurtosis & 115.4271 & 3.6124 \\
\hline Jarque-Bera & 236148.4000 & 49.8829 \\
\hline$p$-value & 0.0000 & 0.0000 \\
\hline Observations & \multicolumn{2}{|l|}{436} \\
\hline
\end{tabular}

Note: Std. Dev. symbolizes the Standard Deviation; $p$-value corresponds to the test of normality based on the Jarque-Bera test.

Table A2. BDS Test

\begin{tabular}{|r|r|r|}
\hline$m$ & $\begin{array}{l}\text { z-statistic of } \\
\text { Residuals of } \\
\text { Equation (2) } \\
\text { with Fixed } \\
\text { Coefficients }\end{array}$ & $p$-value \\
\hline 2 & 6.6405 & 0.0000 \\
\hline 3 & 8.5219 & 0.0000 \\
\hline 4 & 9.5426 & 0.0000 \\
\hline 5 & 9.8704 & 0.0000 \\
\hline 6 & 10.2836 & 0.0000 \\
\hline
\end{tabular}

Note: $m$ stands for the embedded dimension; $p$-value corresponds to the test of i.i.d. residuals based on the $z_{-}^{-}$ statistic of the BDS test. 